\title{
Factors Influencing Health Wearables Adoption and Usage in Saudi Arabia
}

\author{
Mohammed Hokroh \\ School of Management, University of Bolton, United Kingdom
}

\section{Gill Green}

Director of Academic Operations for the Centre for Islamic Finance, University of Bolton, United Kingdom

\section{Mochamad Soleton}

Department of Management, Faculty of Economics and Business, Universitas Mercu Buana, Indonesia

\begin{abstract}
Adoption of technology is one of the most fascinating areas of Management Information Systems (MIS) research. Because it deals with the human kind, adoption of different technologies requires specific case study. Accordingly, the objective of this research study is to explore the factors that influence the adoption and use of wearable health technology in the Kingdom of Saudi Arabia utilizing an extended version of the Technology Acceptance Model (TAM). A total of 104 users were surveyed to test the research hypothesis. Structural Equation Modelling (SEM) was applied to model the research hypotheses and estimate the regressing weights. The research looked at Heart Health (HH), Weight Management (WM), and Sleep Improvement (SI) as antecedents for Perceived Usefulness (PU). Also, Wearable Design (WD), Graphical User Interface (GUI) and Health Information Support (HIS) as antecedents for Perceived Ease of Use (PEU). Findings, limitations and implications are discussed.
\end{abstract}

Keywords: Health Wearables, Management Information Systems (MIS), Saudi Arabia

\section{INTRODUCTION}

According to the World Health Organization (2020) health technology is defined as the application of technology (in the form of devices and systems) to treat health problems and improve the quality of people lives. The increased demand for health-related technology is driven by customers' awareness of health matters. Health motivation is becoming more integrated into people daily routines. Therefore, customers who adopt a "wellness-oriented" lifestyle are more likely to follow more prone health behaviors like regular exercise, diet and use of health technologies. Moreover, wearable technology is paving the ground for the next wave of 
Information and Communications Technology (ICT) revolution. This ICT revolution encouraged manufactures to produce fitness wearables, smartwatches and devices that foster faster and safer health care prevention. Moreover, this ICT revolution helped improve medical practices and accesses to various health information systems at relatively lower cost. The term "healthtology" is becoming more popular referring to the integration of information technology in healthcare to provide innovative solutions to improve overall health awareness and prevention (Dehghani (2018). Accordingly, the objective of this research study is to explore the factors that influence the adoption of wearable health devices in the Kingdom of Saudi Arabia utilizing an extended version of the Technology Acceptance Model (TAM). As pointed out by Chen et al. (2015) wearable health technology aids the development of healthcare sector, enhances the quality of medical service informationization and enables remote medical services utilization. As are result, this research focuses on Heart Health (HH), Weight Management (WM) and Sleep Improvement (SI) as antecedents that promote the usage of health wearables. Moreover, this research looks at Wearable Design (WD), Graphical User Interface (GUI) and Health Information Support (HS) as antecedents that influence the ease of use for health wearables devices. Accordingly, the objective of this research study is to explore the factors that influence the adoption and use of wearable health technology in the Kingdom of Saudi Arabia utilizing an extended version of the Technology Acceptance Model (TAM).

\section{LITERATURE REVIEW:}

\section{The Technology Acceptance Model (TAM):}

Developed by Davis (1986), the Technology Acceptance Model or TAM is considered a predominant theory in the field of technology acceptance. The aims of the theory is to enhance the understanding of user acceptance through in-depth analysis of the design and implementation of an information system. Moreover, to establish a theoretical framework that helps designers and implementers evaluate new systems prior to implementation. The TAM has two main constructs namely Perceived Usefulness (PU) and Perceived Ease of Use (PEU). PU is the degree to which a user believes that using a particular system would enhance his or her job performance while PEU refers to the degree to which a person believes that using a system would be free of effort. Additional external variables can be added to the model and assumed to either influence PEU or PU. Eventfully all variables are assumed to influence the Attitude Toward Use (ATU) which in return influence the Behavioral Intention (BI) and Actual Use (AU) of the system. The simplicity and ease of utilization makes the TAM a popular framework for technology adoption research. TAM has been used in Saudi Arabia to explore system adoption in various fields. For example, Hokroh and Green (2019) investigated the factors that influence video games technology adoption in Saudi Arabia. They surveyed a total of 106 gamers using an extended form of the TAM and found that both PEU and PU are of significant influence to the attitude toward use. Al-Harbi (2011) surveyed 531students to examine the factors that affect e-learning in Saudi Arabia by combining both the TAM and the Theory of Planned Behavior (TPB) and found that self-efficacy and internet experience both significantly influence the PEU. Al-Gahtani (2011) examined electronic web transaction acceptance using an extended form of the TAM and surveyed 128 Saudi users and found that PU had a positive effect on the intention of using online transactions. Likewise, PEU of a website had positively influenced on-line transactions. AlSomali et al. (2009) studied the factors that encouraged customer to adopt online banking in Saudi Arabia. They surveyed a sample of 202 Saudis and found that the quality of the internet connection and self-efficacy both had positive impact on customer PEU of online banking. Social influence and awareness of the services had positive effect on PU. Resistance to change, trust and education level had significant effect on ATU of online banking services. By using the TAM, AlGahtani (2008) investigated the factors that influences computer usage in Saudi Arabia. He 
surveyed a total of 722 voluntary computer end users in 56 private and public organizations in Saudi Arabia. He concluded that behavioural intention positively influences computer usage. ATU and PU positively affect BI to use computers. Also PU and PEU influence ATU. PEU has positive effect on PU.

\section{Health Wearables:}

Davlyatov et al. (2019) examined the correlation between age and the use of Health Information Technology (HIT) in the United States. They studied a national sample of 982 Federally Qualified Health Centers (FQHCS) from 2011 to 2016 and found that as age increased, 4 percent increase in both process and outcome measures of clinical performance are observed annually. More specifically, there is 7 percent higher clinical performance on hypertension control annually in FQHCs which have fully adopted HIT. Through the application of netnography, Dehghani (2018) found that "healthtology" is one of the main factors that influenced users' intentions to adopt smartwatches technology. Zhang et al. (2017) studied the factors that influence healthcare wearable devices in China using the TAM. They randomly surveyed a total of 436 people and found that the adoption intention of healthcare wearable technology is influenced by health, technical and consumer attributes. According to Zhang et al. (2017), health attributes are identified as key factors influencing consumers' adoption of wearable technology and are driven by Health Belief Model (HBM). The HBM is developed by US social psychologists in the Public Health Service to understand why people do not take part in prevention and early disease detection programs. The HBM is also used to predict acute or chronic patients' behavior at the start of treatment program. The HBM has four main constructs which are perceived susceptibility (patients' perception of having a medical condition or contracting a disease), perceived severity (how serious is the health problem is perceived to be), perceived benefits (the personal judgment of the benefits of alternative treatments available that could address the perceived susceptibility and perceived severity) and perceived barriers (negative consequence of a health action that an individual considers taking). Kim and Shin (2015) investigated the psychological determinants of smartwatch adoption in South Korea using an extended version of the TAM. Kim and Shin (2015) surveyed 363 smartwatch users and found that affective quality and relative advantage of smartwatches are associated with PU, while the sense of mobility and availability of smartwatches are associated with PEU. Moreover, the devices' subcultural appeal and cost are found to be antecedents of user attitude and intention of use. Chuah et al. (2016) combined the TAM with psychology to investigate smartwatches adoption in Malaysia. They surveyed 226 users and found that PU and visibility are important factors that drive the intention for use. Hsiaoa and Chenb (2018) explored the factors that influence users' intention to purchase Apple smartwatches in Taiwan through a survey of 260 users. They found that smartwatches design aesthetics, attitude toward and purchase and use are among the most important elements that influence purchasing and using smart Apple watches. Kalantari (2017) conducted a comprehensive synthesis of the literature of wearable technologies consumers' adoption. She found that PU, PEU, price value, perceived enjoyment, visibility, compatibility and design aesthetics are major factors that influence wearable devices adoption. Kalantari and Rauschnabel (2018) conducted a study to explore the factors that influence the adoption of Augmented Reality Smart Glasses (ARSG) by surveying 160 USA students using the TAM. They found that PU, PEU, descriptive norms and image are significantly correlated with the adoption intention.

\section{The Research Theoretical Framework:}

ul Amin et al. (2015) pointed out that the wearable technology started to be used heavily in the medical field since the year 2014. At the beginning it aimed to provide assistance to the medical staff in the operation rooms through providing real-time access to patients' electronic records. The use of these wearable devices has been extended to cover a wide variety of areas including 
personnel health tracking such as blood pressure, pulse and heart rhythm, body posture, walking activity, running and other applications. These applications are become parts of consumer attributes toward using these health wearable technologies (Zhang et al. (2017)). Using an extended version of the TAM, this research aims to unveil the influence of Heart Health $(\mathrm{HH})$, Weight Management (WM), Sleep Improvement (SI), Wearable Design (WD), Graphical User Interface (GUI) and Health Information Support (HIS) on the adoption of health wearable technologies. The findings revealed that $\mathrm{HH}, \mathrm{WM}$ and SI are of a significant importance for the PU of health wearable. Also, WD, GUI and HIS were found to be significant factors influencing the PEU. PU is impacted slightly by PEU and the latter had higher impact on ATU compared to PU. Also, BI is influenced more by ATU compared to PU due to the impact of PEU weight on ATU.

\section{Heart Health $(\mathrm{HH})$ :}

Heart rate monitoring on a regular basis is stated to have a significant positive impact on the health of patients, Phan et al. (2015). Driven by prior literature of Zhang et al. (2017), heart health monitoring is identified as a key factor that influenced consumers' adoption of wearable technology. Wearable heart health features can help users monitor their heart rate as well as their heart rhythm. These features help health aware consumers in detecting and monitoring their heart health regularly. As pointed out by Isakadze and Martin (2019), "incorporating this feature in everyday life of consumers may help raise awareness for $A F$ and facilitate health promotion and preventative efforts". Therefore, the hypothesis this that:

\section{H1: Heart Health $(\mathrm{HH})$ monitoring features positively influence the PU of health wearables.}

\section{Weight Management (WM):}

Weight tracking and management is very important to avoid obesity which is a major cause of disease and mortality worldwide. One innovative way to track and manage weight is through the utilization of wearable devices such as smartwatches and fitness bands. According to a study conducted by Fawcett et al. (2020), wearable devices such as smartwatches and fitness bands can improve the long-term weight loss outcomes by tracking physical activity. As a result, the hypothesis this that:

\section{H2: Weight Management (WM) features positively influence the PU of health wearables.}

\section{Sleep Improvement (SI):}

As pointed out by Shelgikar et al. (2016) the growing consumption of sleep tracking technologies including wearable bands has created new opportunities for collaboration among medical researchers to improve people's health outcomes. For example, smartwatches enabled Rosenberger et al. (2016), to study patients' 24-hour activity cycle of sleep, sedentary, light intensity physical activity in correlation to vigorous physical activities. Sleep self-tracking encourages personal empowerment and customization of application that can lead to sleep improvement and thus overall health enhancement. Users may engage in sleep tracking when there is a perceived personal lifestyle benefits. Moreover, users may feel more encouraged to use wearable technologies if they perceive a positive outcome that could aid their treatment such as improving sleep period or habits. As such it is hypothesized that:

H3: Sleep Improvement (SI) features positively influence the perceived ease of use of health wearables.

\section{Wearable Design (WD):}


The design of health wearables plays a major role in their adoption and use. Kim and Shin (2015) found that wearables appeal is found to be one of the antecedents of user attitude and intention for use. Chuah et al. (2016) found that smartwatches perceived visibility is a factor that derived their use. The term "visibility" is given to define people believes of the extent to which their wearable technology is noticed by others. According to Chuah et al. (2016) warbles such as smartwatches are viewed as a combination of fashion and technology "fashnology". Hsiaoa and Chenb (2018) found that smartwatches design is among the most important elements that influence their purchase and use. According to Kim and Shin (2015) smartwatches are perceived as "aesthetic items" that reflect individual values and characters. The more user friendly the wearable design is the more it is likely to influence adoption. Therefore, the hypothesis is that:

\section{H4: Wearable Design (WD) positively influences the PEU of health wearables.}

\section{Graphical User Interface (GUI):}

The literature points out to the criticality of the Graphical User Interface (GUI) as an important antecedent of applications' adoption and use. According to Agarwal and Prasad (1999) GUI provides capabilities to the users to navigate through an application easily and exchange information between other applications. Al-Mamary et al. (2013) linked GUI to the overall system quality and quality of information. Therefore, information systems that have a user-friendly interface enables users to easily comprehend them and use the systems information more effectively. Therefore, it is assumed that:

\section{H5: Graphical User Interface (GUI) positively influences the PEU of health wearables.}

\section{Health Information Support (HIS):}

The use of health wearable devices can benefit the medical field by capture of patients' information. They can enable doctors to advance both the assessment and counseling in clinical settings and support clinical intervention. For example, in cardiovascular disease prevention, wearable technologies have shown promising results in risk prevention (Lobelo et al. (2016)). However, there is lack of research in how wearables information support influences its adoption and use. In information systems research, user support is found to be of great importance to the adoption process. Hokroh and Green (2018) found that user support had a significant positive role in influencing the adoption and use of information systems. As such the hypothesis is that:

\section{H6: Health Information Support (HIS) positively influences the PEU of health wearables. METHODOLOGY}

The sample of this study was health wearable users (e.g. smartwatches and fitness bands) in the Eastern Province of Saudi Arabia. The data was collected through an online survey questionnaire. Due to the COVID-19 pandemic, participants were randomly recruited via the social communication application (whatsapp). Whatsapp is an application that allows users to share information which may include documents, audios, videos or hyperlinks. The survey hyperlink allowed participants to enter a web page which has the survey questionnaire that requires an average of 10 minutes or less to complete. Before completing the survey, participants can view the objective of survey to get their consent before they take part of the survey.

After removing incomplete and duplicates responses, a total of 104 valid surveys were obtained and analyzed. The study constructs were measured using validated multi-item scales adapted from a previous study of Hokroh and Green (2019). The survey instrument of the questionnaire was modified for the purposes of this research study. A five-point Likert scale was used for all items ranging from "strongly disagree" (1) to "strongly agree" (5). Table-1 shows the demographics of respondents: 
TABLE-1: STUDY DEMOGRAPHICS

\begin{tabular}{|c|c|c|c|c|c|}
\hline & & High School & Undergraduate & Postgraduate & Total \\
\hline \multicolumn{2}{|c|}{ Females } & 14 & 23 & 5 & 42 \\
\hline \multirow{4}{*}{$\begin{array}{l}\text { Age } \\
\text { Group }\end{array}$} & $18-25$ & 11 & 4 & & 15 \\
\hline & $26-35$ & & 15 & 3 & 18 \\
\hline & $36-45$ & & 3 & 2 & 5 \\
\hline & $46-55$ & 3 & 1 & & 4 \\
\hline \multicolumn{2}{|c|}{ Males } & 10 & 48 & 4 & 62 \\
\hline \multirow{5}{*}{$\begin{array}{l}\text { Age } \\
\text { Group }\end{array}$} & $18-25$ & 1 & 6 & & 7 \\
\hline & $26-35$ & 1 & 25 & & 26 \\
\hline & $36-45$ & 2 & 12 & 4 & 18 \\
\hline & $46-55$ & 2 & 5 & & 7 \\
\hline & Above 55 & 4 & & & 4 \\
\hline \multicolumn{2}{|c|}{10} & 24 & 71 & 9 & 104 \\
\hline
\end{tabular}

DATA ANALYSIS:

The reliability analysis outcome is a Cronbach's $\alpha$ value of 0.740 which compatible with earlier studies in the literature (Hokroh and Green (2018) and Sarli and Baharun (2016)). The correlation analysis (Table-2) revealed a significant relationship between Age and Hearth Health (HH) as well as Age and Weight Management (WM). Thus, as people aged the importance of $\mathrm{HH}$ and $\mathrm{WM}$ as antecedents for health wearable devices adoption increased. This indicates that participants' awareness of the importance of WM for $\mathrm{HH}$ is very high and these two features are very critical in determining the PU of health wearables. These findings are compatible with Davlyatov et al. (2019) who found strong correlation between age and the use of Health Information Technology (HIT) in the United States. On the other hand there is a significant negative correlation between Age and Wearable Design (WD). Thus, the WD as antecedent for adoption becomes less important for older users. Although linked to the PEU, the data analysis indicates that younger users may put more importance to the WD compared to older uses. This confirms previous findings on the link between "fashnology" and user (Chuah et al. (2016)). HH is also associated with SI as antecedent for PU. Medical studies associate $\mathrm{HH}$ with getting enough sleep (Rosenberger et al. (2016)). The correlation data indicates that users may be aware of this association and importance. Both WM and SI are associated with WD, GUI and HIS. Thus, as WD, GUI and HIS applications become easier to use, the more importance they become as antecedents for wearables adoption and use. The WD, GUI and HIS are important factors for users PEU of health wearables and they enhance users' adoption of wearables as their application and design become more user-friendly. Gender has no significant correlation with any adoption antecedents.

TABLE-2: CORRELATIONS

\begin{tabular}{|c|ccccccccc|}
\hline & Gender & Age & Education & HH & WM & SI & WD & GUI & HIS \\
\hline Gender & 1 & & & & & & & & \\
Age & $-.303^{* *}$ & 1 & & & & & & & \\
Education & -.106 & -.016 & 1 & & & & & & \\
HH & -.037 & $.257^{* *}$ & -.014 & 1 & & & & \\
WM & .085 & $.209^{*}$ & .045 & $.428^{* *}$ & 1 & & & &
\end{tabular}




\begin{tabular}{|c|c|c|c|c|c|c|c|c|c|}
\hline SI & .123 & .068 & .085 & $.393^{* *}$ & $.612^{* *}$ & 1 & & & \\
\hline WD & .176 & $-.228^{*}$ & .167 & .053 & $.241^{*}$ & $.364^{* *}$ & 1 & & \\
\hline GUI & .124 & -.056 & -.091 & .016 & $.338^{* *}$ & $.218^{*}$ & $.546^{* *}$ & 1 & \\
\hline HIS & .069 & .117 & .009 & .010 & $.345^{* *}$ & $.293^{* *}$ & $.611^{* *}$ & $.487^{* *}$ & 1 \\
\hline
\end{tabular}

**Correlation is significant at the 0.01 level (2-tailed).

*Correlation is significant at the 0.05 level (2-tailed).

$N=104$

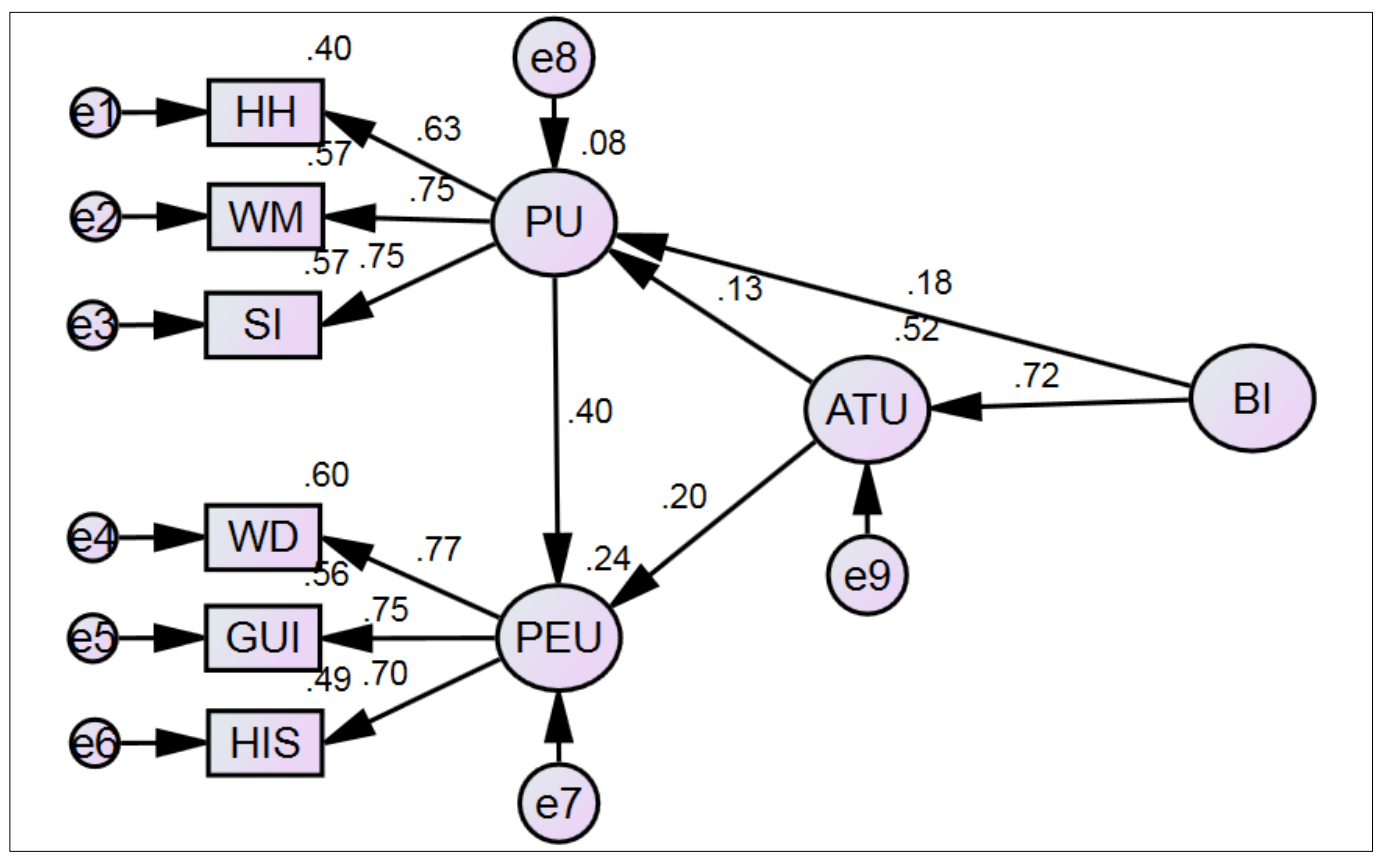

Figure 1: PATh ANALYsis

Structural Equation Modelling (SEM) using AMOS-23 was applied to develop to model the research hypotheses and estimate the regressing weights through confirmatory factor analysis. The path model revealed that $\mathrm{HH}, \mathrm{WM}$ and SI are of a significant importance for the PU of health wearable devices supporting H1, H2 and H3. However, WM and SI had high regression weights compared to $\mathrm{HH}(0.75$ vs. 0.63$)$ indicating that participants associate more importance to these factors to PU. This may be the result of the association between $\mathrm{HH}$ and WM and SI. Thus, if a wearable can help users manage their weight and improve sleep, then they might have better $\mathrm{HH}$. Therefore, WM and SI become more critical in determining health wearables PU. On the other hand, the path model also revealed a strong association between WD, GUI and HIS as factors influencing the PEU with regression weights of $0.77,0.75$ and 0.70 respectively supporting $\mathrm{H} 4, \mathrm{H} 5$ and $\mathrm{H} 6$. WD had the strongest association with the PEU followed by GUI. Users associate a user-friendly design and application with the ease of use of the health wearables. Also, having a user-friendly HIS is of critical importance to users as they getting a feedback on their health status. PU is impacted slightly by PEU and the latter had higher impact on ATU compared to PU. This means that users put more importance on fashionable and user friendly wearable for adoption compared to its functions. Also, BI is influenced more by ATU compared to PU due to the impact of PEU weight on ATU.

DISCUSSION AND LIMITATIONS: 
The study is aimed at exploring the factors that influence the adoption of wearable health devices in the Kingdom of Saudi Arabia. The findings revealed a strong association between HH, WM and SI and the PU of health wearable. Also, WD, GUI and HIS were found to be significant factors influencing the PEU. There is an association between PU and PEU and the latter had higher impact on ATU compared to PU. BI is influenced more by ATU compared to PU. However, this study had limitations and implications. The study sample was taken randomly from the Eastern Province community of Saudi Arabia. Thus, future studies may assess this research finding by looking at larger sample or conducting a longitudinal study to confirm findings. On the other hand, a critical importance has to be given to the issue of information privacy especially with HIS. Silva et al. (2020) highlighted the criticality of information privacy in relation to mobile health application. With the recent developing of mobile devices, wearables and applications, a new perspective on health care applications is growing. Therefore, ensuring patients' information privacy becomes challenging in order to strike a balance between devices adoption and information protection. Future research may look and address these challenges.

\section{CONCLUSION}

The objective of this research study is to explore the factors that influence the adoption of wearable health devices in the Kingdom of Saudi Arabia utilizing an extended version of the Technology Acceptance Model (TAM). A total of 104 users were surveyed to test the research hypothesis. Structural Equation Modelling (SEM) was applied to model the research hypotheses and estimate the regressing weights. The findings revealed that $\mathrm{HH}, \mathrm{WM}$ and SI are of a significant importance for the PU of health wearable. Also, WD, GUI and HIS were found to be significant factors influencing the PEU. PU is impacted slightly by PEU and the latter had higher impact on ATU compared to PU. Also, BI is influenced more by ATU compared to PU due to the impact of PEU weight on ATU.

\section{REFERENCES}

Agarwal, R. and Prasad, J. (1999) 'Are individual differences germane to the acceptance of new information technologies?' Decision sciences, 30(2), 361-391.

Al-Gahtani, S. (2008) 'Testing for the Applicability of the TAM Model in the Arabic Context: Exploring an Extended TAM with Three Moderating Factors' Information Resource Management 21, 1-26. 
Al-Gahtani, S. S. (2011) 'Modelling the Electronic Transaction Acceptance Using an Extended Technology Acceptance Model' Applied Computing and Informatics 9, 47-77.

Al-Harbi, K. (2011) ‘E-learning in the Saudi Tertiary Education: Potential and Challenges’ Applied Computing and Informatics 9, 31-46.

Al-Mamary, Y. H., Shamsuddin, A., Hamid, A., \& Aziati, N. (2013) 'the impact of management information systems adoption in managerial decision making: A review' Management Information Systems, 8(4), 010-017.

Al-Somali, S. A., Gholami, R. and Clegg, B. (2009) 'An Investigation into the Acceptance of Online Banking in Saudi Arabia' Technovation 29, 130-141.

Chen, M., Zhang, Y., Li, Y., Hassan, M. M., and Alamri, A. (2015) 'AIWAC: Affective interaction through wearable computing and cloud technology' IEEE Wireless Communications, 22(1), 20-27.

Chuah, S. H. W., Rauschnabel, P. A., Krey, N., Nguyen, B., Ramayah, T. and Lade, S. (2016) 'Wearable technologies: The role of usefulness and visibility in smartwatch adoption' Computers in Human Behavior 65, 276-284.

Davis, F. D. (1986) 'A Technology Acceptance Model for Empirically Testing New End-User Information Systems: Theory and Results' Doctoral Dissertation, Salon School of Management Cambridge, MA.

Davlyatov, G., Borkowski, N., Feldman, S., Qu, H., Burke, D., Bronstein, J. and Brickman, A. (2019) 'Health Information Technology Adoption and Clinical Performance in Federally Qualified Health Centers' Journal for healthcare quality: official publication of the National Association for Healthcare Quality.

Dawi, N. M. and Jalil, N. A. (2019, November) Integrated model for smartwatch adoption. In Proceedings of the International Conference on Advanced Information Science and System (pp. 1-7).

Dehghani, M. (2018) 'Exploring the motivational factors on continuous usage intention of smartwatches among actual users' Behaviour \& Information Technology, 37(2), 145-158.

Fawcett, E., Van Velthoven, M. H and Meinert, E (2020) ‘Long-Term Weight Management Using Wearable Technology in Overweight and Obese Adults: Systematic Review' JMIR mHealth and uHealth, 8(3), e13461.

Hokroh, M., \& Green, G. (2018) 'System adoption: socio-technical integration' International Journal of Business Management and Technology, 2(5), 95-107.

Hokroh, M., and Green, G. (2019) 'Online Video Games Adoption: Toward an Online Game Adoption Model', International Journal of Research in Business and Social Science 8(4) 163171.

Hsiao, K. L. and Chen, C. C. (2016) 'What drives in-app purchase intention for mobile games? An examination of perceived values and loyalty' Electronic Commerce Research and Applications, 16, 18-29.

Isakadze, N. and Martin, S. S. (2019) 'How useful is the smartwatch ECG?' Trends in cardiovascular medicine.

Kalantari, M. and Rauschnabel, P. (2018) 'Exploring the Early Adopters of Augmented Reality Smart Glasses: The Case of Microsoft HoloLens' Augmented Reality and Virtual Reality, 229-245. Cham: Springer 
Kalantari, M. (2017) 'Consumers' adoption of wearable technologies: literature review, synthesis, and future research agenda' International Journal of Technology Marketing, 12(3), 274-307.

Kim, K. J. and Shin, D. H. (2015) 'An acceptance model for smart watches' Internet Research 25(4) 527541.

Lobelo, F., Kelli, H. M., Tejedor, S. C., Pratt, M., McConnell, M. V., Martin, S. S., \& Welk, G. J. (2016) 'The wild wild west: a framework to integrate mHealth software applications and wearables to support physical activity assessment, counseling and interventions for cardiovascular disease risk reduction' Progress in cardiovascular diseases, 58(6), 584-594.

Phan, D., Siong, L. Y., Pathirana, P. N., \& Seneviratne, A. (2015) 'Smartwatch: Performance evaluation for long-term heart rate monitoring' In 2015 International symposium on bioelectronics and bioinformatics (ISBB) (pp. 144-147). IEEE.

Rosenberger, M. E., Buman, M. P., Haskell, W. L., McConnell, M. V., \& Carstensen, L. L. (2016) '24 hours of sleep, sedentary behavior, and physical activity with nine wearable devices' Medicine and science in sports and exercise, 48(3), 457.

Sarli, A. and Baharun, R. (2016) Modelling and Statistical Concepts in Business Research: AMOS and SPSS, Lexington, KY: USA.

Shelgikar, A. V., Anderson, P. F., \& Stephens, M. R. (2016) 'Sleep tracking, wearable technology, and opportunities for research and clinical care' Chest, 150(3), 732-743.

Silva, W., Sacramento, C., Silva, E., Garcia, A.C. and Leal Ferreira, S.B. (2020, January) Health Information, Human Factors and Privacy Issues in Mobile Health Applications. In Proceedings of the 53rd Hawaii International Conference on System Sciences

ul Amin, R., Inayat, I., \& Shazad, B. (2015, April) ‘Wearable learning technology: A smart way to teach elementary school students', 2015 12th Learning and Technology Conference (pp. 1-5).

World Health Organization (2020) Health technology assessment [online] Available at: https://www.who.int/health-technology-assessment/about/healthtechnology/en/ [Accessed 14 June 2020].

Zhang, M., M. Luo, R. Nie, and Y. Zhang (2017) “Technical Attributes, Health Attribute, Consumer Attributes and Their Roles in Adoption Intention of Healthcare Wearable Technology." International Journal of Medical Informatics 108: 97-109. 